Historic, Archive Document

Do not assume content reflects current scientific knowledge, policies, or practices. 



\subsection{7}
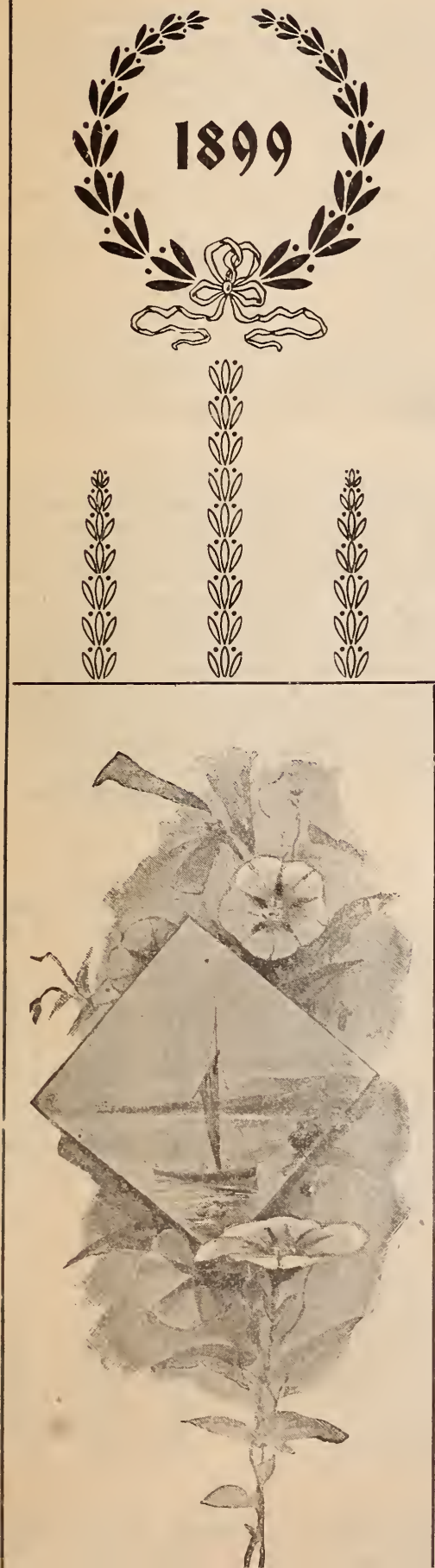

1., sive \&:

HEB 281899

77im. Brinker's

SPECIAL

oo Rlpice Uic

FOR

market Gardeners. AND

Florists. D2

329 PROSPECT STREET, OPP. GRAY'S ARIMORY.

$\stackrel{*}{*}$

Cslepbone 2349. 
INSTRUCTIONS TO PURCHASERS.
V. E are prepared to execute orders immediately after this Catalogue is issued, and it will be to the advantage of purchasers as well as to us, if they send in their orders at as early a date as possible. Do not delay ordering until such time as you wish to use the seeds

PACKING. No charge for boxes, bags or barrels on vegetable seeds, bulbs or potatoes.

All Seed, both Vegetable and Flower, before we send them out, are tested as to vitality and to as great an extend as possible as to genuiness.

WARRANTY. We warrant that all Seeds sold by us shall prove to be as represented, to this extent, that should they prove otherwise, we will replace them, or send other seeds to the same value.

t. we cannot guarantee the crop any further than the above offer, as there are so many ' $r$ which we have no control, which operate unfavorably in the germination of seeds ity of the crop. Among the causes of failure may be mentioved unfavorable hich is one of the most important. The soil may be in proper condition when the lanted, but the weather which follows may be cold and wet, which will cause the ot ; or it may be hot and dey, which destroys the germ before it shows itself. The seeu, may be, and are frequently, destroyed by vermin of various kinds. Such occurences are beyond the power of man to prevent, and for them we cannot be responsible. We wish it understood, therefure, that while wa exercise the greatest care to have all seeds pure and reliable, we do not give any warranty, expressed or implied, further than above stated. If the purchaser does not accept the seeds on these terms and conditions, they must be returned at once, and the money that has been paid for them will be refunded. Wishing every recipient of this list good crops, and good prices for everything they raise in '99, I remain,

Yours truly,

WM. BRINKER.

Oz. $1 / 4$ lb. Lb.

ASPARAGUS, Palmetto \$0 $05 \$ \$ 015 \quad \$ 050$

Culossal...................... $5 \quad 10 \quad 30$

Columbian Ma m moth

White.....

$10 \quad 25$

\section{ASPARAGUS ROOTS-}

Conovers Colossal, 2 yrs. old, per $1000, \$ 500$

Barrs Mammoth, "6 "6 " " 500

Palmetto.............. 66 "6 6 500

Qt. Pk. Bus.

BEA NS, BUSH, Wax Podded-

Grolden Wax................ \$0 $25 \quad \$ 100 \quad \$ 375$

Wardwell's Kidney Wax

$\begin{array}{lllll}30 & 1 & 50 & 5 & 50\end{array}$

German Black Wax......

Currie's Rustproof Wax

$25 \quad 110$

$25 \quad 110 \quad 4 \quad 25$

Refugee Wax

$\begin{array}{lllll}25 & 1 & 10 & 4 & 10\end{array}$

Yospmite M a moth Wrax.

Davis Kidney Wax.......

$\begin{array}{lllll}40 & 1 & 75 & 750\end{array}$

๑5 $120 \quad 425$

BEA NS, BUSHFD, Green Podded-

Burpee Bush Lima......

Improved R॰d Vulentine

Extra Early $R$ of ug e $\theta$ 1,000 to 1

$30 \quad 160$

$25 \quad 125$

600

25110

475

BEETS-

Yellow Six Weeks.........

Qt.

Brown Six Weeks, Mohawk.. ................

Emperor William ..........

Large White Kidney.... "6 "6 Marrow....

Refugee on 1,000 to $1 \ldots$.

Canadian Wonder.........

Hor ticultural $\mathrm{D}$ warf......

Dreer's Bush Lima.......

New Stringless Lima.....

Giant Green Podded Stringless Valentine...

$25 \quad 110 \quad 400$

$\begin{array}{lllll}25 & 1 & 10 & 4 & 00\end{array}$

$25 \quad 120 \quad 450$

$20 \quad 80 \quad 300$

$\begin{array}{llll}20 & 80 & 3 & 00\end{array}$

$25 \quad 120 \quad 425$

$\begin{array}{lllll}25 & 1 & 20 & 4 & 25\end{array}$

$\begin{array}{lllll}20 & 1 & 10 & 4 & 00\end{array}$

$\begin{array}{lllll}35 & 2 & 00 & 7 & 50\end{array}$

$\begin{array}{lll}30 & 150 & 500\end{array}$

$\begin{array}{llll}40 & 200 & 750\end{array}$

BEANS, Pole or Running-

Ex. Large Lima............

King of the Garden, Lima ......................

Dutch Case Knife..........

Kent'kv Wonder............

Lazy Wife....

Scarlet Runner

30

550

Crosby Improved.

Oz. $1 / 4 \mathrm{lb}$.

$\$ 020$

Lb.

Egyptian .....................

New Columbia 
BEETS-Continued.

Mitchell's Red Turnip...

Lenz's Early B lood Turnip....

Beckerts Winter.........

Detroit Blood Red.........

Eclipse Blrod Turnip...

Dewing's Blnod Turnip..

Edmund's Blnod Turnip

Long Blnod Red.

Swiss Chard

BEETS, Mangel-

Mrmmoth Long Red.....

Golden Turkard

Norbition Giant.............

Velmoreus Improved.....

BROCCOLI-

White Cape.

BRUSSELS SPROUTS-

Improved Half Dwarf...
Oz. $1 / 4 \mathrm{lb}, \mathrm{Lb}$.

$\begin{array}{lll}5 & 15 & 40\end{array}$

$5 \quad 15 \quad 40$

$10 \quad 20 \quad 60$

$10 \quad 20 \quad 50$

$\begin{array}{lll}5 & 15 & 40\end{array}$

15

15

15

20

40

10

40

60

10

$\begin{array}{lll}5 & 15 & 30 \\ 5 & 15 & 30 \\ 5 & 15 & 30 \\ 5 & 20 & 50\end{array}$

CABBAGE-

Ex.Ear. Jersey Wakefi'd

Large Jersey Wakefield or Charleston............

Henderson's Ear. Summer

All Seasons or Vandergaw ......................

Surehead

Select Late Flat Dutch.

Burpee all Head...........

Autumn King or World

Beater.

Late Drumhead

Perfection Drumbead Savoy.....................

Red Dutch..................

Mammoth Red Rock.....

Danish Ball Head Winter

Early Dwarf Ulm Savoy

Fotter's Improved

Brunswick ...............

Early Flat Dutch..........

New Ideal...................

Lauderback's all Year Round

Market Gardener's Cab-

bage No. 2...............

CRESS, Curled..

Nitive or Upland ......... 100

Water.

20

20

60

200

15

60

230

15

15

15

15

15

15

15

15

20

25

15

15

15

15

20

40

150

40

40

40

150

150

50

140

160

40

150

40

140

40

40

140

140

$50 \quad 175$

$\begin{array}{lll}70 & 2 & 25\end{array}$

$40 \quad 150$

$40 \quad 140$

$40 \quad 150$

-40 150

60

225

30

85

300

CORN SALAD -

Large-Leaved.

Large Rooted.

$10 \quad 20$

70 。
CAULIFLOWER, Early-

Gilt Edge Early Snowball..

Extra Early Erfurt.......

Extra Early Paris.........

Algiers ......................

Lenormand's ShortStem
Half-Early Paris (Nonpariml) .

Autumn Giant.

\& 20

20

10

10

10

$1 \mathrm{Oz}$

$4 \mathrm{Oz}$.

CELERY, White Plume (Selected Stock)......... \$

Golden Self-Blanching..

Giant $\mathrm{Pa}=\mathrm{c}$.................

Boston Market..

New Rose

B 'ckert's Hardy Winter

Perle Le Grand ..........

Golden Heart Giant.....

$\begin{array}{llll}10 & 60 & 2 & 00\end{array}$

$10 \quad 40 \quad 150$

Oz. $1 / 4 \mathrm{lb}$. $\mathrm{Lb}$.

$20 \$ 60 \quad \$ 200$

$\begin{array}{lll}25 & 75 & 250\end{array}$

$15 \quad 45 \quad 150$

$\begin{array}{lll}15 & 40 & 150\end{array}$

$\begin{array}{llll}20 & 60 & 2 & 00\end{array}$

$\begin{array}{lllll}30 & 1 & 25 & 5 & 00\end{array}$

$\begin{array}{llll}20 & 60 & 2 & 25\end{array}$

$15 \quad 50 \quad 150$

CELERI:AC-

Prugue Giant.............. $\quad 15 \quad 40 \quad 150$

Apple Shaped

$15 \quad 40 \quad 135$

\section{CARROT-}

Half-Long Point-Rooted $\quad 10 \quad 20 \quad 60$

Chantany .................. $10 \quad 20 \quad 60$

Danvers Half-Long....... $10 \quad 20 \quad 60$

Rubicon....................... $10 \quad 25 \quad 70$

$\begin{array}{llll}\text { Improved Long Orange. } & 10 & 20 & 60\end{array}$

Carentan :................. $10 \quad 20 \quad 60$

Guerande or Oxhart...... $10 . \quad 20 \quad 60$

Half-Large Nantes........ $10 \quad 20 \quad 60$

Large White Belgian..... $5 \quad 15 \quad 35$

Large Yellow Belgian... $\quad 5 \quad 15 \quad 35$

Early Scarlet Hnrn....... $10 \quad 20 \quad 60$

Coreless Long Red....... $10 \quad 20 \quad 60$

\section{CUCUM BERS-}

Forcing Wn. Spine.......

Bennott's Wh. Spine.....

Imp. White Spine.........

Long Green ...............

Jersey Pickle ..............

Livingston Evergreen...

Everbearing ................

Chicago Pickle.............

Boston Pickle

Small Gherkin or Burr.

Ex. Long White Spine..

Evergreen White Spine.

Early Russian.

Early Cluster ..............

Rolleson's Filegraph...

15

50

150

5

15

15

15

15

15

15

15

15

20

20

20

20

50

50

50

50

50

50

50

50

60

50

50

10

10

Pkt. 25c

CORN, Sugar-

Early White Cory

Qt.

Eırly Minnesota..

$\$ 015$

Pk. Bus.

Early Crosby

15

Perry's Hybrid.

Russell's Prolific.

$\$ 075 \quad \$ 275$

$\begin{array}{ll}75 & 275\end{array}$

$\begin{array}{lll}75 & 2 & 75\end{array}$

$75 \quad 275$ 
CORN-Continued.

Early Mammoth...........

Stowell's Evergreen......

Late Mammoth............

Country Gentleman......

Mammoth White Cory.

Ferry's Evergreen..........

Landreth Sugar ...........

Kendal Early Giant......

Black Mexican.

DANDELION-

Improved Thick Leaved \$

Oz. 1/4 lb. Lb

$40 \quad \$ 125 \quad \$ 400$

\section{EGG-PLANT-}

New York Spineless......

ENDIVES, Green Curled

Broad-Leaved.

Moss Curled.

\section{KOHLRABI-}

Wh. Very Early Vienna

Purple Very Er. Vienna

\section{KALE-}

Curled MossbackWinter

Dwarf German Greens

(Siberian)

Early Moss Curled.........

\section{IETI UCE-}

Black Seeded Simpson..

Beckert's Golden Curled

Perpignan, or Defiance..

Grand Rapids.

California Cream Butter

Big Boston..................

Hanson . ......................

All Year Round...........

New Silver Ball...........

New Sensation..............

Meyer's All Right.........

New York Cabbage.......

Salamander ....................

Deacon Head.

Tennis Ball...................

Frankford Head White Seed

Frankford Head Black

Seed.

\section{LEEK-}

London Flag

New Giant Italian

Carentan
25

20

20

$70 \quad 250$

15

15

15

15

5

5
MUSK MELONSOz. 1/4lb. Lb. Emerald Gem ........... 10 \$ 10 \$ 50 $\begin{array}{llll}\text { Extra Eurly Hackensack } & 10 & 20 & 50\end{array}$ Chicago Market........... $10 \quad 20 \quad 50$ Surprise .................... $10 \quad 20 \quad 50$ Prolific Nutmeg............. $10 \quad 20 \quad 50$ Miller's Cream or Osage $10 \quad 20 \quad 50$ Winter Musk .............. $10 \quad 20 . \quad 50$ Rocky Ford................ $15 \quad 40 \quad 100$ Paul Rose.................. $15 \quad 35 \quad 125$ Hackensack, Turk's Cap $\quad \begin{array}{lll}10 & 20 & 50\end{array}$ Jenny Lird................. $10 \quad 20 \quad 50$ Golden Netted Gem..... $10 \quad 20 \quad 50$ Orange Christian......... $10 \quad 15 \quad 50$ Shumway's Giant......... $10 \quad 15 \quad 50$ Tip Top ...................... 10 Pine Apple................. 10

MELONS, W ATER-

Kolb's Gem ...............

Semidole ....................

Florida Favorite.............

Ruby Gold....

Boss.

....................

10

10

10

10

GreenCitron(RedSeeded)

10

NASTURTIUM-

Tall $\$ 010$

Dwarf..

10

$\$ 030 \quad \$ 100$

$\begin{array}{lll}30 & 1 & 00\end{array}$

\section{ONIONS-}

Yellow Globe Danvers (Extra Select) ...........

Yellow Danvers(for sets)

Strasburg, or Yellow Dutch ....................

Southport Whité Globe

Prizetaker ..................

Red Wethersfield..........

White Portugal Silver Skin

$20 \quad 50$

$20 \quad 50$

$20 \quad 50$

2050

$20 \quad 50$

$20 \quad 50$

Early Barletta...........

Ohio Globe Danvers......

Bloomsdale Early Pearl

Bloomsdale Gold Seal...

White Pickling.

\section{ONION SETS-}

White Sets........................ \$0 25

Qt. Bus. Mk. Pr.

Yellow Sets. 25

Multiplier 25

OKRAOz. $1 / 4 \mathrm{lb}$. Lb.

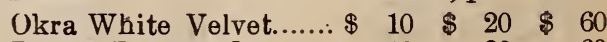
Dwarf Improved.......... $10 \$ 20 \$ 60$

PEPPER, Bull Nose..... $\quad 20 \quad 60 \quad 200$ Ruby King .................. $\quad 20 \quad 60 \quad 200$

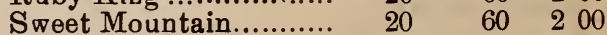
Imp. Long Red ............. $20 \quad 60 \quad 200$ Lg. Red Cayenne........ 20 - 60200 
PEPPER-Continued.

Procop Giant.

Red Cherry

Red Chili.

\section{PARSLEY-}

Champion Moss Curled.

Plain Leaved

Hamburger T u r n i p Rooted

\section{PARSNIPS-}

Imp. Hollow Crown......

$\begin{array}{rrr}\text { Oz. } & 1 / 4 \text { lb. } & \text { Lb. } \\ 20 & 60 & 200 \\ 20 & 60 & 200 \\ 20 & 60 & 200\end{array}$

10

10

10

$$
20
$$

15

15

60

50

50

\section{PUMPKIN-}

Large Cheere.............

Mammoth King............

Connecticut Field..........

Cushaw, or Crockneck..

Golden Oblong. ............

Quaker Pie....

Sweet or Sugar..............

Calhoun....

Tennessee Sweet Potato

PEAS-

Alaska

First and Best (Extra select stock) .............

Landreths Extra Early.

Maud S.

Nott's Excelsior... .... ...

McLean's Advancer......

Dwarf Champion..........

Telephone ..................

Pride of the Market......

Juno

Horsford Market Garden

Heroine...... .............

Shropshire Hero . ..........

Sharp's New Queen......

Duke of Albany .. .........

Champion of England...

White-Eyed Marrowfat, (hand picked)

Black-Eyed Marrowfat, (hand picked.)

10

10

5

10

10

10

10

10

10

Qt. Pk. Bus. $\$ 020$ \$0 75

20

25

20

25

20

25

25

25

25

25

25

25

25

25

20

15

15

75

100

75

110

90

110

125

125

110

180

100

125

125

125

80

$$
60
$$

60

Oz. $1 / 4 \mathrm{lb}$

RADISH-

$\$ 010$

E. White Box

10

Cincinnati Market........

New Glass.................. tra Long Scarlet).......

Vick's Scarlet Globe.......

Livingston New Pearl...

Burpee's Surprise.........

Rapid Forcings, or Rosy Gem

Early Dęep Red Turnip

Early Scarlet Globe......

French Breakfast.........

Wood's Early Frame.....

350

350

400

350

300

175

$\mathrm{Lb}$.

$\$ 0 \quad 15 \quad \$ 050$

$20 \quad 60$

$\begin{array}{lll}10 & 20 & 60\end{array}$

10

10

$20 \quad 50$

$\begin{array}{lll}10 & 15 & 50\end{array}$

$10 \quad 20 \quad 60$

$10 \quad 15$

50

$10 \quad 15$

$10 \quad 15$

$10 \quad 15$

$10 \quad 15$
RADISH-Continued.

Beckert's I mproved Chartier..

Beckert's Nonpareil, or White Chartier..........

Beckert's Chartier Turnips ......................

Lnng White Strasburg...

White Vienna (Lady

Finger) ..................

Long Scarlet Short Top

Celestial ......................

Golden Summer.............

Gray Summer Turnip...

Long Black Spanish......

Round Black Spanish...

Chinese Rose Winter.....

Improved Golden Bale..

Giant Stuttgart............

New Laafless...............

White Summer Turnip..

Oz. $\quad 1 / 4 \mathrm{lb} \quad \mathrm{Lb}$.

$10 \quad 15$

50

$10 \quad 15$

50

$10 \quad 15 \quad 50$

$10 \quad 15 \quad 50$

$10 \quad 15 \quad 50$

$10 \quad 15 \quad 50$

$10 \quad 15 \quad 50$

$10 \quad 15 \quad 50$

$10 \quad 15 \quad 50$

$10 \quad 15 \quad 50$

$10 \quad 15 \quad 50$

$10 \quad 15 \quad 50$

$10 \quad 15 \quad 50$

$10 \quad 15 \quad 50$

$25 \quad 75 \quad 250$

$10 \quad 15 \quad 50$

\section{SQUASH-}

W. Bush Scalloped.......

Prolific Marrow....

Golden Custard............

Boston Marrow .............

Hubhard

Marblehead...

Golden Summer Crookneck ......................

Yellow Bush Scalloped..

Fordhook ..................

Chicago Warted Hub bard.

10

15

50

10

10

10

10

10

10

10

10

20

60

\section{SALSIFY -}

Mammoth Sand wi ch Island

10

35

125

SPINACH-

Thick Leafed, Long Standing ...............

Bloomsdale, 1899 Crop.

Norfolk Savoy ..............

Beckert's Hardy Winter

La. Rd. Thick Leafed...

Victoria

TUR NIPS-

Jersey Lily.

Purple Top Strap-Leafed

Purple Tup Globe.........

White Dutch ..............

White Egg..

Yellow Aberdeen...........

Yeliow Stone..............

Improved Golden Bale...

Early White Flat Dutch

Purple Top Milan.........

Early White Milan.........

Oz. $1 / 4 \mathrm{lb} . \mathrm{Lb}$.

$\$ 0 \quad 10 \quad \$ 0 \quad 20 \quad \$ 0 \quad 60$

$\$ 200 \quad \$ 0 \quad 25$

$250 \quad 30$

$200 \quad 25$

$200 \quad 30$

$200 \quad 25$

$200 \quad 25$

RUTA-BAGA, or SWEDISH TURNIP-

Imp. American............ 5 15 40

Sweet German............. $\quad 5 \quad 15 \quad 40$ 
TOMATO-

Atlantic Prize

Yellow Plumb

Dwarf Champion

Acme

Beauty

The Stone

Ponderosa

Money Maker

Early Bird

Dwarf Aristocrat

Lorellard

Yellow Plumb
Oz. $1 / 4 \mathrm{lb} . \mathrm{Lb}$.

$\begin{array}{llll}15 & 40 & 150\end{array}$

$\begin{array}{lll}15 & 40 & 150\end{array}$

$\begin{array}{llll}15 & 40 & 150\end{array}$

$\begin{array}{lll}15 & 40 & 150\end{array}$

$\begin{array}{lll}15 & 40 & 150\end{array}$

15

30

25

30

15

20

15

00350

$45 \quad 150$

$\begin{array}{lll}60 & 200\end{array}$

$50 \quad 150$

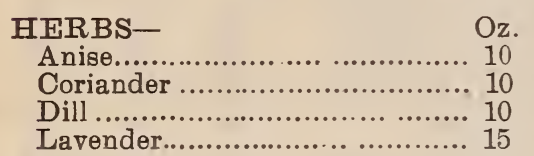

HERBS-Continued.

Oz. Lb.

Sage

Sweet Basil

20

20

20

Sweet Marjoram

15

Thyme

20

Qt. Pkt. Bus.

\section{FIELD CORN-}

Leaming.

Pride of the North

$10 \quad 40 \quad \$ 125$

POP CORN-

White Rice.

Golden Queen

Lb.

$\$ 70$

50

50
150
FIELD PEAS-

Canada Field.

. Cow Peas.
$\mathrm{Lb}$

$5 c$

150

140

150

100

275

$5 \mathrm{c}$
Qt. Pkt. Bus.

$10 \quad 40 \quad \$ 125$

$\begin{array}{llll}10 & 40 & 1 & 25\end{array}$

\section{BIRD SEED.}

Mixed Canary.....

Canary, Best Sicily.

Hemp

Rape.
$8 \mathrm{c} . \mathrm{lb}$.

8c." ",

8c. "“

$8 c$. "

\section{Millet.}

Sunflower Seed

Maw or Poppy S-ed

Lettuce Seeds for Birds. 8c. lb. $8 c . " 6$ $15 \mathrm{c}$." " 25̃c. "

\section{CHOICE NORTHERN GROWN SEED POTATOES.}

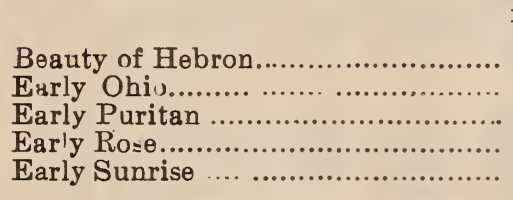

GRASS Sit ED.

Timothy

Red Top.

Kentucky Blue Grass

Orchard Grass.

Sweet Bernal.

\section{MARKET}

PRICE.

State of Maine

Green M untain.

The Freeman...

Irish Cobbler

Empire State

\section{CLOVER SEED.}

Common Red

Mammoth nr Large Red

Scarlet or Crimson

Alsike $\cap r$ Swedish

White Dutch 


\section{FLORISTS' FLOWER SEEDS.}

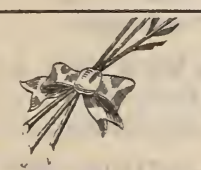

We devote equally as much care and attention to the department of florists' seeds as to vegetable seeds for market gardeners. Our stocks are grown for us by the best growers and specialists, both at home and abroad, and entire confidence can be placed in our strains herewith offered, as it has always been our policy to secure the very best obtainable, cost being a secondary consideration. We would specially recommend our strains of Aster, Calceolaria, Cineraria, Pansy, Petunia, Primula, Stocks, Verbena, Cyclamen, Cosmos, Mignonette, and all such other seeds as are much in demand by florists.

\section{All Flower Seeds are Tested for Vitality Before Being Sent Out}

\begin{tabular}{cccc}
\multicolumn{3}{c}{ Pkt. } & Oz. \\
ABUTILON, choice mixed ...... 50 & 5 & \\
& & & \\
AgERATUM, Little Dorrit, & & \\
Dwarf Blue........................... & 5 & 75 \\
White.............................. & 5 & 60
\end{tabular}

ARALIA Sisbaldi

50

ASTER Improved Victoria Flowers very large, beautifully imbricated and perfect in form; excellent for cutting, or as a pot plant.

Pure White...

Rusy Carmine............................

P.each Blossom....................... 10

Crimson............................... 10

Azure Blue.......................... 10

Dark Blue............................ 10

Mixed............................. 10

Aster, Comet. Flowers very large, composed of long, wavy, twisted petals, arranged in loose yet dense half-globes, resembling Japanese.

Pure White.......................... 10

Rose ................................. 10

Crimson................................... 10

Light Blue................................. 10

Mixed
Pkt. Oz.

Aster, Queen of the Market. Extremely early; three woeks earliar than other varieties.

Pure White.......................... $10 \quad 150$

Carmine............................ $10 \quad 150$

Light Blue ............................ $10 \quad 150$

Rose ................................. $10 \quad 150$

Mixed.................................. 1010

Aster, Pæony-Flowered Perfection. Very double, large, and of fine shape, petals beauti fully incurved.

Pure White........................... $10 \quad 200$

Brilliant Rose ............................ $10 \quad 200$

Crimson ............................. $10 \quad 200$

Light Blue ....................... $10 \quad 200$

Mixed................................ $10 \quad 150$

Aster, Mignon. Resembling the Victoria race, but still more floriferous. The flowers are of refined form, and keep fresh a long time. Mixed.............. $10 \quad 200$

Aster, Vick's Branching. Flow ers pure white, of good size, long stems and broad, long petals

Aster, Dwarf Bouquet. Mixed.. $10 \quad 150$ 
ASTER-Continued.

Pkt.

Aster, Dwarf Chryeanthemum.

Mired.

Aster, Semple's Branching. Plants of branching habit, producing on long stems flowers of perfect form, from 4 to 6 inches in diameter.

White.

Lavender............................., 10

Pink .................................. 10

Mixed.

ALYSSUM, Little Gem. Grows but 4 inches high ; pure white ; very dwarf and free-flowering.. Maritimum, Sweet............... Saxatile, compactum

ANTIRRHINUM. Tall mixed. Dwarf mixed

AQUILEGIA. Gold Spurred..... Grandifloro alba.................... Vulgaris, Double Mixed ..... ...

ACROCLI NIUM, Double Rose. Double White.

AMPELOPIS Veitchii..lb., $\$ 1.50$

AMMOBIUM alatum.

AURICULA, Fine Mixed.........

BALSA M,Florists'Double White

Camellia-flowered, Mixed......... colors

Double Mixed.

BEGONIA, Tuberous-rooted,

Double..................50c. and $\$ 100$

Tuberour-rooted, Single 25c and 50

Vernon................................. 25

New Giant .......................... 50

Dwarf Vernon....................... 25

Vulcan.

BELLIS PFRENNIS, (Double Daisy.) Our strain of this important florists' flower is of unsurpassed quality.

Mammoth Mixtd.................. 10

Longfellow, Rose.................... 10

Snowball, White....................... 10

Double Mixed.

400

300

300

300

BROWALLIA elata

CALCFOLARIA, Giant Strain, Superb Mixed...........50c and $\$ 100$

CINERARIA, hybrida grandiflora. Our giant strain of cineraria is unsurpassed in size of flowers or beauty of colors. Florists desiring the best Cineraria obtainable should try this......................50c and $\$ 100$ Cineraria Maritima................ 5

50
Oz.

CALENDULA, Orange King.. Prince of Orange. Striped; very fine.

Pkt. Oz.

5

CALLIOPSIS, Golden Wave.... Tinctora.

COREOPSIS, lanceolata grandiflora. Very large, golden yellow flowers; excellent perennial. .

Sunbeams.

CAMPANULA Media. (Canterbury Bell)..........................
Calycanthema (Cup and Saucer)

CA NDYTUFT, Empress......... Tom Thumb..

30

White Rocket..............................

Carmine.

Crimson.................................

Flesh

Mixed

CANNA, New Dwarf, French Choice Mixed......................

CARNATIONS, Semi-Dwarf Marguerite. Choicest Mixed. This new class has justified highest expectation. Seedings begin blooming the fuurth month from time of coming up, producing large and very sweet scented flowers: about 80 per cent. of them come double and produce a rich display of colors.

Striped

Striped.................................... 10

150

Rure White.

Rose.....

Scarlet.

Early Dwarf Vienna................

Grenadine. Early Dwarf.........

Perpetual or Tree....................

Double Self Colors .....................

Hardy Double......................... , 10

CENTAUREA candidissima.... 10

Gymnocarpa.......................... Cyanus Emperor William.......... " Mixed

Marguerite.

20

20

50

20

Chameleon

COB开A scandens.

40

COLEUS, Choice Mired

50

15

10

COSMOS

Mammoth Perfection Erlinda, Snowy White......................

Mammoth Perfection Rosita, Beautiful Pink...................... Mammoth Perfection Conchita, Crimson Velvet........................ New Dwarf Dawn......................

Extra Early............................. 10

Giant Early Flowering, Mixed.. 10 
Pkt. Oz.

CELOSIA. Dwarf Yellow

10

Empress, Dwarf Scarlet............ 10

Glaagow Prize (President Thiers) Crimson.................... 10

Japonica .............................. 5

Plumosa, Mixed....................... 5

Ostrich Feather..................... 10

Rosea .................................. 10

CONVOLVULUS Majur..Ib 60c

New Jupanese.........................

Minor

Mauritanicus.

Major fl. pl...

CYPRESS VIi E, Mixed

CYCLAMEN, California Giants.

1,000 seeds. 100 seeds.

Fine Mixed........\$8 $00 \quad \$ 100$

White .............. $800 \quad 100$

White,red center $800 \quad 100$

Crimson............ $800 \quad 100$

Pink ................. $800 \quad 100$

Rose ................. $800 \quad 100$

Persicum, Fine Mixed ............. 30

DAHLI A S, Single Mired........... 5

Double Mixed ....................... 10

Lilliput, Mixed..................... 10

DELPHINIUM, formosum...... 5

Cashmeriana ....................... 10

Tall Rocket, Mixed .................. 5

Dwarf Rocket, Mixed............. 5

DATURA, cornucopia............ 10

DIGITALIS, Mixed............... 5

DIANTHUS Chinensis Double

Mixed ..............................

Chinensis, Double White.........

Diadematus, $\mathrm{fl} . \mathrm{pl}$...................

Imperialis, fl. pl.....................

Heddewigii grandiflora, fl. pl.
(Crown of Perfection)

“ Eastern Queen.........

" Crimson Belle.

$\begin{array}{lll} & 5 & 50 \\ & \end{array}$

Barbatus(Sweet William) Single

66 $6{ }_{66}{ }_{60}$ Double

EUCALYPTUS globulus

EUPHORBIA heterophylia.....

ESCHSCHOL ZIA............... 5

FEVERFEW, Double White... 5

GAILLARDIA grandiflora...... 5

Lorenziana

Single Mixed

GLOXINIA hybrida grandiflora.

GOURDS, many sorts mixed......
Pkt. Oz.

GREVILLEA robusta............ 5 50

GLOBE AMARANTH, Mixed 540

OOLETIA, Finest Mixed......... 5

HELICHRYSUM, Mixed........ 5

HOLLYHOCK, Double White;

best...............................

Double Pink ; best...................

Double Red; best...................

Double Yellow ; best................ 5

Double Mixed; best................. . 5

Allegheny..............1/8 oz., \$1.25 25

HUMUIUS Japonica .............. $5 \quad 30$

Japonica variegata................... 5

ICE PLANT....................... 5 30

IMPATIENS Sultana......5 and 25

IPOMGEA (Moonflower)............ 5 40

Bona nox............................. 5

Setosa ........................................ 5

Heavenly Blue....................... $10 \quad 125$

"Northern Light..................... 5.40

KENILWORTH IVY ......... 5

LANTANA, Mixed................ 5

30

LOBELI A erecta Crystal Palace compacta. True Deep Blue;

fine ................................ 10

Erinus speciosa Superba (Trailing) Blue with white eye........

Erinus Crystal Palace...............

MARVEL OF PERU ........... 5

MARIGOLD, Eldorado........... 5

Tall French................... . 5

Dwarf French........................ 5

300

100

75

15

25

20

20

MAURANDIA, Mixed........... 5

MUSK.

5

MIMULUS tigrinus grandiflora.

5

MUSA ensete (Abvesinian Banana).........per 100 seeds $\$ 1.25$.

MIMOSA pudica (Sensitive Plant)................................. 5

MIGNONETTE,Defiance (New) 10

Machet................................ 5

Golden Queen............................ 5

Victoria, Dark Red.................... 5

Parsons; White........................ 5

Mile's Spiral............................. 5

Gabriella............................ 5

Large Flowering Common, lb.75c.

MYOSOTIS, Eliza Fonrobert.... 10

Victoria. Best for pots: blue... 10

"6 Rose....................... 10

Alpestris. Blue....................... 15

Palustris. Dark Blue.............. 10 
NASTURTIUM, Tall, Choice Mixed......................lb. $\$ 1.00$

Oz.

NASTURTIUM Tall, Hybrids Mad Gunter......................... Tall, Superb Mixed.................

Dwarf, Choice M'xed....... $\$ 1.25$

Beruty, Yellow and Scarlet....................

Empress of India. Brilliant Crimson...............

Golden King, Golden Yèllow ....................

King Theodore. Dark Scarlet....................

Aurora. Silmon, Rose . and Blush............... Cloth of Gold. Scarlet, gold foliage..............

Prince Henry. Cream Spotted Crimson.........

Crystal Palace Gem......

NICOTIANA affinis.

OXALIS tropæoloides.

\section{PHLOX DRUMMONDII,}

Fringed...

Star of Quedlinburg.

Grandiflora Varieties:-

Scarlet Pure White.

Striped, Mixed

Chamois-Rose

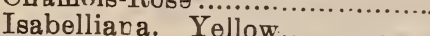

Coccinea. Scarlet.

Kermesina. Scarlet, white eye...

Violacea. Purple, white eye......

Atropurpurea. Purplish red....

Alba oculata. White claret eye.

Choicest Mixed.

Nana compacta, Choire Mixed..

Perennial. Choicest Mixed......

Perennial Dwarf, Choicest Mixed.

PORTULACA. Single Mixed....

Double Grandiflora.

PYRETHRUM, aureum (Golden Feather)

PYRETHRUM Roseum hybridum fl. pl.

PYRETHRUM Roseum hybridum Single.

POPPY, Tulip.

Shirley .................................

Danebrog

Mikado

White Swan ............................

Pæony-flowered.

Oriental
PRIMULA

Pkt. Oz.

Choicest Chinese Fringed-

Bright Red...............50 and 100

Spotted, White and Crimson.................50 and 100

Striped...................50 and 100

Crimson. Yellow eye...50 and 100

Alba magnifica..........50 and 100

Rr se ....................50 and 100

Blue. White eye......50 and 100

Fringed, Choicest

Mixed...................50 and 100

Fern-lesved .............50 and 100

Double White............50 and 100

Choicest Mixed...........50 and 100

Obconica........................... 25

Grandiflora......................... 50

Elatior ................................ 25

J в ponica...........................

Vulgaris .............................. , , 10

PETUNIA, Giants of California. A grand strain of Petunia. The large, beautiful, fluffy blossoms are handsomely crimped, fringed and ruffled, and come in many exquisite colors.......................

Large Flowering Fringed, Choicest Mixed................... 50

Large Flowering, Choicest

$$
\begin{aligned}
& \text { " ‘ Yellow throated } \\
& \text { Fine ............. } 25 \\
& \text { " " } \text { "Fringed Double }
\end{aligned}
$$

Inimitable, White and Crimson striped.

Inimitable D warf, Striped ......... Good Mixed.

Striped and Blotched

\section{PANSY-}

Beckert's Superb Mixed ........... 10

\section{Cassier's Very Large Five} Blotched

Bugnot's Superb Mixed............

Odier Five-Blotched.................

Giant Golden Five-Spotted ....... "6 Parisian. Large Stained.. " Yellow, Black eye; fine.. " Striped.

" B Blue. Emperor Willia.....

"، Black ...........................

"6 White Purple eye.........

"6 Atropurpurea Dark purple...

100

10

"Beaconsfield. Deep purplish violet, top petal a white hue............... 
Pkt. Oz.

RICINUS Zanzibariensis. New, Borboniensis ....

Gibsoni

Cambodgensis

SALVIA splendens. Scarlet.... 10

Patens. Blue ....................... 10

- Compacta. A dense compactgrowing form of the preceding

SEDUMS. Cœruleum, Glaucum, Maximowiczii, Telephium and Mixed 10

SMILAX.

STOCKS. Our strains of German Ten Weeks Stocks are vary fine, a large percentage of them coming double.

Large-flowering Ten Weeks Pure White..........................

Flesh Color.

Best Yellow ...............................

Scarlet

Dark Blood Red

Light Blue

Pink ....

Dark Blue.

Choicest Mixed

Improved Snow flake.

Princess Alice. (Cut-and-Come Again) Alice. (Cut and-Come

TORENIA Bailloni. Golden yellow, hrown throat.............

Fourvieri. Sky-blue, yellow center

THUNBERGIA alata. Buff, dark eve........................... Alba. White, dark eye............. Aurantiaca. Orange, dark eye.. Bokeri. Pure white.

Mixed

TROPGOLUM Lobbianum..... Canariensis. Canary Creeper....

VERBENA. Our strain is the best ever offered. Large, elegant flowers, colors clear and beautiful, with many prettily variegat ${ }^{\circ}$ and daintily marked.

Mammoth White

Puirple

P.nk.

Scarlet..................

Choicest Mixed ....... \\ 200}

VIOLA odorata, White.

Odora!a, Blue.........................

WALLFLOWER, Double Mixed................................. 10

Single Mixed..........................

ZIN NIA, grandiflora, Mixed . ... 5

Elegans, Mixed........................ 5

Lilliput .................................

Curled and Crest ${ }^{2}$....................

40

Fireball.

$\begin{array}{rr}10 & \\ 5 & \\ 5 & 50 \\ 5 & 30 \\ 5 & 60 \\ 5 & 75 \\ & 30\end{array}$

SWEET PEAS,

Price, all Varieties, ounce 10 cents; $1 / 4$ pound 20 cents; pound 75 cents.

Blanch Burpee, New White.

California Light Pink.

Blushing Batuty, Soft Pink.

Katherins Tracy, Finest Pink.

Blanche Ferry, Pink and White.

Butterfly. White, Edged Blue.

Cardina1. Cardinal crimson.

Captain of the Blues. Purplish claret, wings blue.

Countess of Radnor. Delicate lavender.

Emily Exford. Redish mauve.

Emily Herderson. Pure white.

Empress of India. Rosy pink, wings blush white.

Fireflv. Fiery scarlet, wings crimson scarlet.

Her Majesty. Deep rosy crimson.

Mrs. Gladstone. Soft blush, suffused pink.

Mrs. Sankey. Large white flowers, changing to blush.

Orange Prince. Orange Salmon, with bright pink wings.

Queen of the Isle. Scarlet, white stripes, mottled.

Venus Salmon. Buff.

Stanley. Dark Maroone.

Cupid. Packet 5 cents.

Superb Mixture.

\section{0}

60

30 


\section{INSECTICIDES.}

Slug shot, per pound 5 cents.

Parisgreen, per pound 30 cents.

Tobacco dust, 10 pounds 35 cents.

Spray pumps, $\$ 300$.

Nicotina, per can, $\$ 1.50$.

Antipest, quart 85 cents.

Flowers of sulpher, pound 5 cents.

Powdered hellebore, pound 25 cents.

Fostite, pound 10 cents.

Fostite bellows, \$3.50.

Rat and mouse biscuit, box 25 cents.

Ant exterminator, boz 20 cents.

Roach exterminator, box 20 cent. .

Pure raw bone, per $100, \$ 2.00$.

Bradley lawn fertilizer, 10 pounds, 50 cente.

Bradley plant food, box 15 cents.

Sheep manure, per $100, \$ 2.50$.

Peruvian guano, per $100, \$ 2.50$.

Cleveland lawn weeder, 50 cents.

\section{SPRING BULBS.}

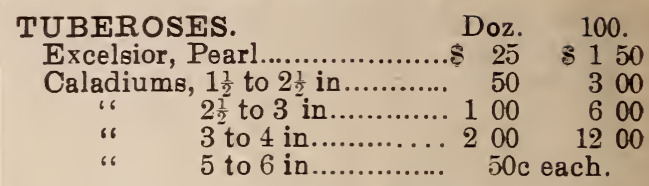

GLADIOLUS, (Cushman's)... 25

Callas Rıchardia.................. 100

Cinnamon vine.................... $1 \mathrm{CO}$

Maderia vine ..................... 50

Spider lilies........................ 150

Lilium auratum .................... 150

roseum.................. $150 \quad 1000$

album ...................... $150 \quad 1000$

150

600

We shall in season have a large and select line of all bulbs adspted for florists' use.

Freesias. Mammoth size. June and Juıy.

Lilium Harrisii. July and August.

Koman Hyacinths. August and September.

Lily Candidum and Paper White Grandi-

flora. September and October.

Dutch Hyacinths, Tulips and Narcissus. September and October.

Lilies.-Chinese Narcissus. October and November.

Address

WM. BRINKER,

329 Prospect St., Opp. Gray's Armory, CLEVELAND, 0.

Telephone, Main 2349. 